\title{
Assessment of Pediatric First Permanent Molar Treatment under General Anesthesia at the King Abdulaziz Medical City, Jeddah, Saudi Arabia
}

\author{
Raniah Baakdah, Alanoud Alhelali, Asrar Sindi, Ebtesam Aldegail, and Ranin Ba-Shikh
}

\section{ABSTRACT}

Background: The use of dental general anesthesia (GA) among children has seen an increase globally, but there are consequences in using GA in children, which include changes in the treatment plan. First permanent molar (FPM) treatment under GA is very strategic, but there is a lack of studies that assess the protocol of FPM treatment under GA. This study determines the type and factors that affect children's FPM treatment under GA at the King Abdulaziz Medical City (KAMC) in Jeddah, Saudi Arabia.

Methods: A cross-sectional study reviewed dental rehabilitation cases in children between the ages of 5 and 14 years old from 2015 to 2018. These children had erupted first permanent molars and were treated under GA.

Results: This study included 394 children, with 1330 FPMs treated under general anesthesia. The frequency of FPM treatments administered were color restoration $(49 \%)$, fissure sealant application $(37 \%)$, SSC $(8.9 \%)$, and amalgam filling (4.5\%). Low pulpal treatment was reported which was mostly involved indirect pulp capping. Most of the final restorations were covering only one surface, followed by the two-surface restorations, and then by SSC. The type of FPMs treatment administered on children was significantly influenced by their ages, gender, and health conditions. The type of tooth restoration and pulp therapy treatments were significantly dependent on children's age.

Conclusions: The pediatric dentists' strategy for FPMs treatment under general anesthesia at KAMC followed AAPD treatment guidelines considering children's age, gender, and health status. The FPM treatment was mostly restorative followed by preventive, and the least treatment was extraction treatments. KAMC pediatric dentist efforts should be directed to initiate more preventive programs and effective dental home care utilization at PHC centers.

Keywords: Dental caries, first permanent molar, general anesthesia, pediatric rehabilitation, extraction, restoration, operation time.
Published Online: January 13, 2022

ISSN: $2684-4443$

DOI : $10.24018 /$ ejdent.2022.3.1.137

\section{R. Baakdah*}

King Abdulaziz Medical City, Jeddah, Saudi Arabia.

(e-mail: baakdar@ hotmail.com)

A. Alhelali

Private Clinic, Jeddah, Saudi Arabia.

(e-mail: anood t@ hotmail.com)

A. Sindi

$\mathrm{MOH}$, Taief, Saudi Arabia.

(e-mail: sososindi3@gmail.com)

E. Aldegail

Private Clinic, Jeddah, Saudi Arabia.

(e-mail: ebtesam_1a1@ hotmail.com)

R. Ba-Shikh

Private Clinic, Jeddah, Saudi Arabia.

(e-mail: dr.raneen77@gmail.com)

*Corresponding Author

\section{INTRODUCTION}

The first permanent molars (FPMs) are the key to occlusion due to their importance in maintaining the teeth' occlusion and keeping the vertical dimension of the face [1]. They are considered the first permanent teeth to form and erupt in the oral cavity of children between 5 to 6 years old. They may last through the lifetime of the individual. Their early formation and longer exposure to the oral environment increase the risks of developing defects or forming caries [2]. Based on a study conducted in Saudi Arabia, 75.5\% of the FPMs had been affected with caries or pulpal pathology [3]. More than $50 \%$ of children over 11 years had some experiences with caries in their FPMs [2]. A Romanian study reported that the frequency of FPMs extraction was between 30 and $40 \%$ among 13- to 14-year-old children, who did not undergo regular dental care [4].
The treatments of decayed FPMs among children may range from applying preventive fluoride or sealant to extraction of the tooth. Generally, the treatments of FPMs among children vary according to the defect of the tooth, the child's cooperation during the treatment, and other factors. With increased public awareness to maintain healthy teeth and control caries' incidences, dentists may consider restoring the FPMs that are affected extensively by caries and exhibit pulpal symptoms during the mixed dentition stage. However, seventy percent $(70 \%)$ of FPM extraction was reported to be due to badly decayed teeth, especially for mandibular FPM [5], [6]. Untreated caries FPMs may experience unfavorable occlusal changes if left unrestored, compared to the effects of its extraction during the mixed dentition stage. Overall, the spontaneous space closure rate ranged from $33.3 \%$ to $94 \%$ in the maxilla and from $50 \%$ to $75 \%$ in the mandible [7]. Pediatric dentists' decision should weigh the risks and the benefits of keeping heavily restored FPMs versus its early 
extraction based on treatment consequences and outcomes [8].

The children dental care was provided for $47.6 \%$ of Saudi children using a pharmacological intervention to provide a good quality dental care [9]-[11]. Children treated under GA were those with extensive dental need, anxiety, or medical condition. General anesthesia is considered a safe practice in children's dental treatment with mild complications, such as those with post-operative pain or those experiencing nausea and vomiting during longer operation times under GA [5], [12]. Majority of pediatric dentists carefully consider the treatment of the FPMs, due to its effect on the operation time and the required operator skills, such as surgical skills, advanced restorative skills, and root canal treatment or other pulp therapy procedures [13]. Although high quality dental treatment is required under GA, long anesthetic duration risk should be avoided. An increase in total dental anesthetic time was significantly increase post-operative complications [14]. Some hospitals recommended 30 minutes as the suitable limit for a day stay under GA. Others preferred a maximum length of 2 hours for the whole procedure on a day care facility [15], [16]. In a Saudi multi-center study, the mean duration of a pediatric dental treatment under GA was determined to be $97.9 \pm 49.9 \mathrm{~min}$, with a range of 5 to $224 \mathrm{~min}$ [13].

In pediatric dentistry literature, no reports were found to describe the dentist's strategy in treating FPMs under GA and the factors that affect the FPM treatment. This study was aimed to determine the frequency and the type of the FPM treatments given to children under GA and to assess the factors that affect the pediatric dentist's decision about FPM treatments under GA at the King Abdulaziz Medical City (KAMC) in Jeddah City.

\section{METHODS}

This retrospective cross-sectional study were data derived from the pediatric dental rehabilitation cases at KAMCJeddah dental department during 2015 to 2018. Pediatric dental section at KAMC-Jeddah is treating freely National Guard children below 14 years of age. Yearly about 250-300 pediatric dental rehabilitation cases performed in the center. A total of 1,038 pediatric dental rehabilitation under general anesthesia records reviewed. The included sample population were children with ages from 5 to 14 years old. Cases with no eruption of the first permanent molars and those with missing records were excluded from this study. The data collected included the demographic profiles of the children, such as their ages, gender, and health status; the backgrounds about their operations, such as the type of their cases, their dental operation times, case sequences, the operators' level of training, the dental services administered; and the FPM treatments, including the methods of treatment, the types of restoration, the treated surfaces, and pulp therapies.

The data were processed using SPSS to derive the means and the standard deviations, and to carry out inferential analyses using the t-test and the Chi-square, where appropriate. P-values less than 0.05 were considered as significant.

\section{RESUlTS}

Our study included 394 records of pediatric dental rehabilitation procedure. Our sample population consisted of 209 (53\%) males and 185 (47\%) females. The mean age was 7.8 years old (1.8 SD), and the median age was 8 years old. Majority of these children, i.e., 276 (72\%), had ages between 5 and 8 years old during the time of this study, and only $5 \%$ of them were above 11 years old. More than half of the children, i.e., 233 (69\%), were healthy, whereas 103 (31\%) of them were described as medically compromised. The demographic profiles of the children are shown in Fig. 1.

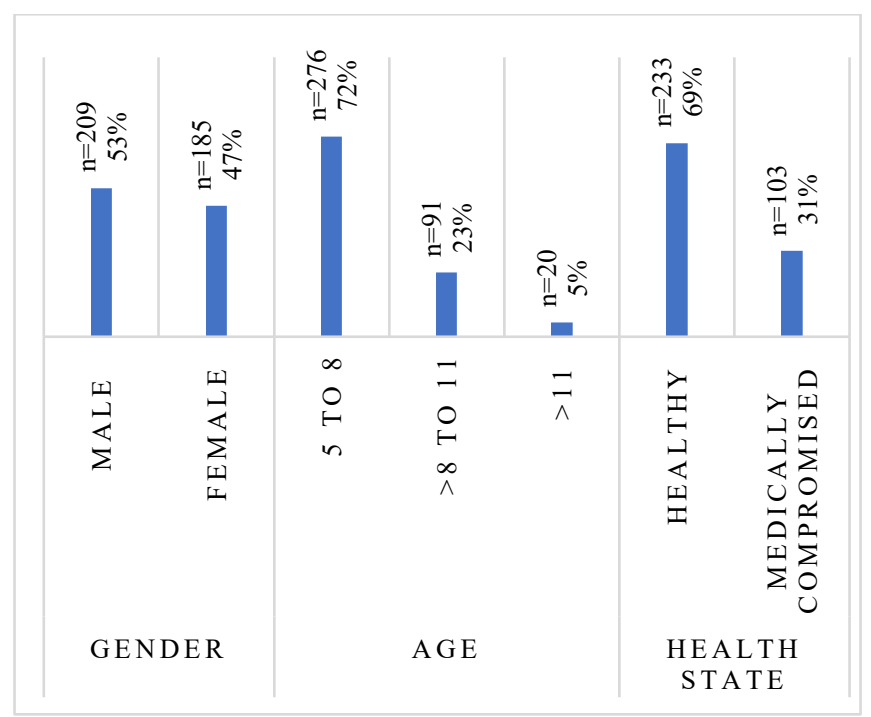

Fig. 1. Demographic data of the patients included in the study.

The general descriptions of the dental procedures performed under GA are shown in Fig. 2. The number of dental procedures completed under GA ranged from 2 to 52 dental procedures. The mean number of dental procedures for each child was 15.4 (4.3 SD), and the median was 15 . There were six cases, where extraction was the only procedure done. Thirteen cases were treated with restoration only, and 375 cases were treated with a combination of extraction and restoration of the primary and permanent teeth under GA. Although all dental rehabilitation under general anesthesia were supervised by a consultant, the operators for 196 cases studied were mostly senior residents. Specialized pediatric dentists handled 164 cases, and junior residents dealt with only 34 cases. The mean surgery time was $120 \mathrm{~min}$, and the median operation time was $115 \mathrm{~min}$. The most common surgery times were between 60 and 120 minutes, which were observed in the treatments of 190 children, followed by 120 to $180 \mathrm{~min}$ with 143 children. Most of the operators were male pediatric dentists. Most treatments, i.e., 268 cases, for the pediatric dental rehabilitation under GA were done in the morning.

In 394 dental rehabilitation cases. treatment of the FPMs can be categorized as, 108 cases $(27 \%)$ preventive treatments, 221 cases $(56 \%)$ restorative, and 65 cases $(17 \%)$ extraction. In 32 cases $(8 \%)$ comprised both restorative and extraction treatments. The FPM extraction under GA was done for all four FPMs in 33 cases, one FPM extraction in 20 cases, two FPMs extraction in 10 cases, and three FPMs extraction in only 2 cases.

The erupted FPMs treatment (total of 1,330 FPM), were 
summarized in Table I. The frequency of the lower FPMs treatment is higher than that of the upper FPMs, but this difference is not statistically significant. The FPM treatments administered consisted of color restoration (49\%), fissure sealant application (37\%), SSC (8.9\%), and amalgam filling (4.5\%). Majority of the FPMs pulpal treatment involved indirect pulp capping (78\%), and only (12.8\%) were for direct pulp capping. Very limited treatment was carried out through pulpotomy or RCT under GA. Most of the final restorations that were done covered only one surface, followed by the two-surface restorations, and then by SSC. Regarding the treatment methods, there were no significant differences between the positions of the FPMs, that is, whether they were of the maxilla or mandible, or the right or the left $(\mathrm{P}=0.86)$. When comparing the types of restoration, the surfaces, and the pulp therapy for the positions of the FPMs, there were also no significant differences that were noted.

The relationship between the demographic data and the treatment methods, as well as therapy of the patients, are shown in Table II. The type of treatment was significantly influenced by the ages of the children $(\mathrm{P}<0.001)$, their gender $(\mathrm{P}<0.0008)$, and their health conditions $(\mathrm{P}<0.0001)$. More preventive and restorative treatments were reported in the younger, male, and healthy children, but a higher number of extractions of the FPMs was reported among the 8-11-yearold group, female, and medically compromised children.

The need for tooth restoration was significantly related to the age factor $(P<0.0001)$ of the children. Significantly higher number of restorations could be related to the younger age group, to fissure sealant $(84.50 \%)$, resin restoration $(69.82 \%)$, amalgam $(54.90 \%)$, and stainless-steel crown (63.0\%). Also, pulp therapy was significantly driven by the ages $(\mathrm{P}<0.0001)$ of the children. Direct or indirect pulp capping and pulpotomy were applied to the children of the younger age group.

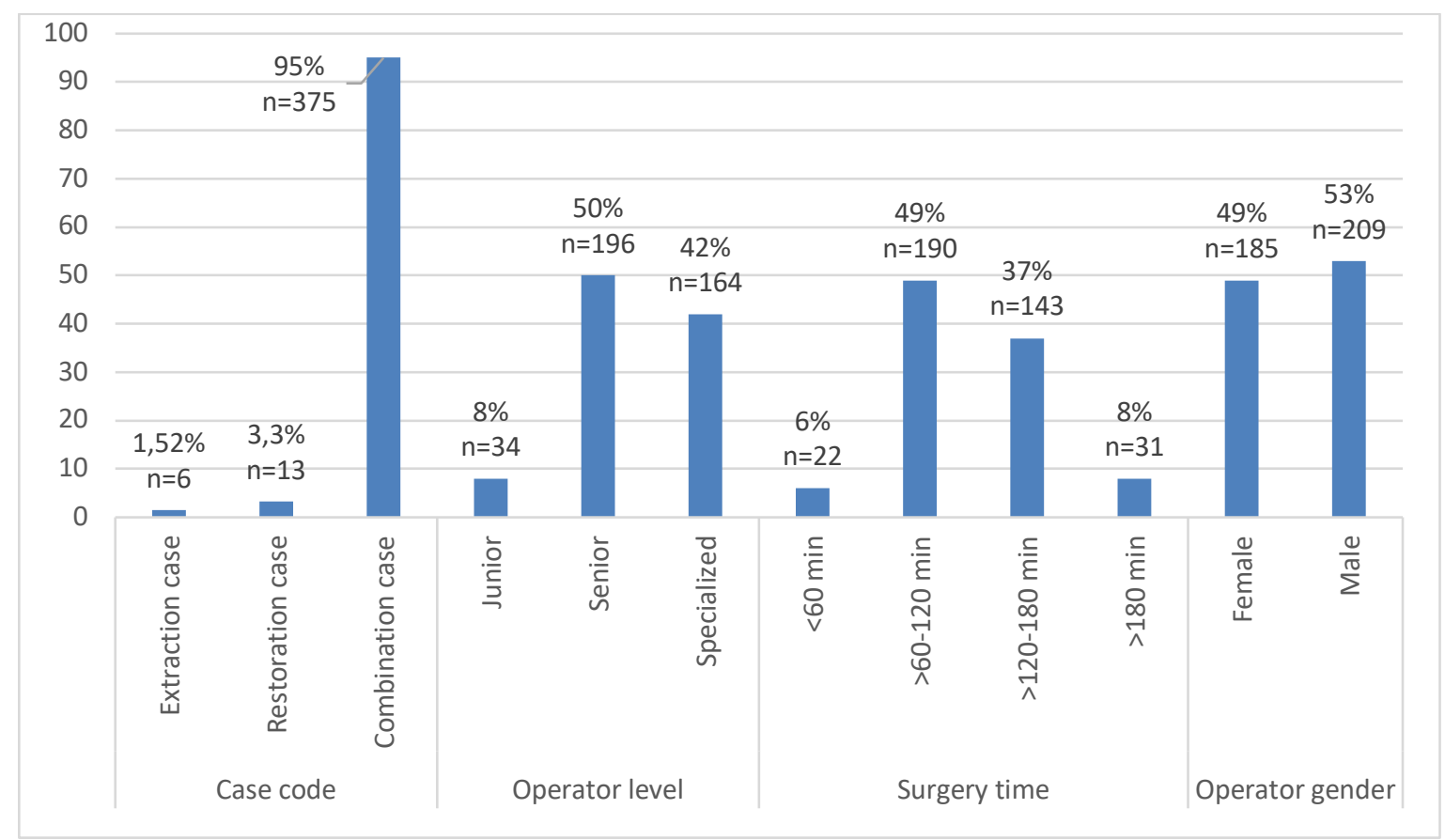

Fig. 2. Frequencies and other data on the pediatric dental rehabilitation procedures in the study.

TABLE I: FREQUENCY OF DENTAL TREATMENT FOR FPMS

\begin{tabular}{|c|c|c|c|c|c|c|c|}
\hline & & 16 & 26 & 36 & 46 & Total & P value \\
\hline \multirow{4}{*}{$\begin{array}{l}\text { Type of } \\
\text { restoration }\end{array}$} & Fissure sealant & $114(41.5 \%)$ & $94(36.2 \%)$ & $108(36.0 \%)$ & $1112(36.1 \%)$ & $42428(37.4 \%)$ & \multirow{4}{*}{0.4479} \\
\hline & Colored restoration & $129(46.9 \%)$ & $138(52.9 \%)$ & $141(47.0 \%)$ & $157(50.7 \%)$ & $565(49.3 \%)$ & \\
\hline & Amalgam & $11(4.0 \%)$ & $7(2.7 \%)$ & $18(6.0 \%)$ & $15(4.9 \%)$ & $51(4.4 \%)$ & \\
\hline & $\mathrm{SSC}$ & $21(7.6 \%)$ & $22(8.4 \%)$ & $33(11.0 \%)$ & $26(8.4 \%)$ & $102(8.9 \%)$ & \\
\hline \multirow{4}{*}{$\begin{array}{l}\text { No. of } \\
\text { restoration } \\
\text { surfaces }\end{array}$} & One surface & $98(60.5 \%)$ & $74(54.4 \%)$ & $1113(60.1 \%)$ & $113(58.6 \%)$ & $398(58.6 \%)$ & \multirow{4}{*}{0.1979} \\
\hline & Two surfaces & $40(24.7 \%)$ & $33(24.3 \%)$ & $38(20.2 \%)$ & $53(27.5 \%)$ & $164(24.2 \%)$ & \\
\hline & Three surfaces & $3(1.9 \%)$ & $7(5.2 \%)$ & $4(2.1 \%)$ & $1(0.5 \%)$ & $15(2.2 \%)$ & \\
\hline & $>3$ surfaces & $21(12.9 \%)$ & $22(16.2 \%)$ & $33(17.6 \%)$ & $26(13.5 \%)$ & $102(15.0 \%)$ & \\
\hline \multirow{4}{*}{$\begin{array}{l}\text { Type of } \\
\text { pulp } \\
\text { therapy }\end{array}$} & Direct pulp capping & $2(13.3 \%)$ & 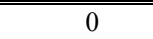 & $2(8.0 \%)$ & $6(21.4 \%)$ & $10(12.8 \%)$ & \multirow{4}{*}{0.6735} \\
\hline & Indirect pulp capping & $11(73.3 \%)$ & $8(80.0 \%)$ & $21(84.0 \%)$ & $21(75.0 \%)$ & $61(78.2 \%)$ & \\
\hline & Pulpotomy & $1(6.7 \%)$ & $1(10.0 \%)$ & $1(4.0 \%)$ & $1(3.6 \%)$ & $4(5.1 \%)$ & \\
\hline & RCT & $1(6.7 \%)$ & $1(10.0 \%)$ & $1(4.0 \%)$ & 0 & $3(3.9 \%)$ & \\
\hline
\end{tabular}


TABLE II: FACTORS THAT AFFECT FPMS TREATMENT UNDER GA

\begin{tabular}{|c|c|c|c|c|c|c|c|c|}
\hline & & \multicolumn{3}{|c|}{ Age } & \multicolumn{2}{|c|}{ Gender } & \multicolumn{2}{|c|}{ Health Status } \\
\hline & & $5-8$ & $>8-11$ & $>11$ & Male & Female & Healthy & $\begin{array}{c}\text { Medically } \\
\text { compromised }\end{array}$ \\
\hline \multirow{4}{*}{$\begin{array}{c}\text { Treatment } \\
\text { Method }\end{array}$} & Preventive & $355(84.9 \%)$ & $60(14.4 \%)$ & $3(0.7 \%)$ & $241(55.8 \%)$ & $191(44.2 \%)$ & $260(68.8 \%)$ & $188(31.2 \%)$ \\
\hline & Restoration & $482(67.6 \%)$ & $190(26.7 \%)$ & $41(5.8 \%)$ & $383(53.2 \%)$ & $337(46.8 \%)$ & $451(72.9 \%)$ & $167(27.0 \%)$ \\
\hline & Extraction & $51(30.4 \%$ & $85(50.6 \%)$ & $32(19.1 \%)$ & $70(39.3 \%)$ & $108(60.8 \%)$ & $60(46.9 \%)$ & $68(53.1 \%)$ \\
\hline & $P$ value & & $<0.001 *$ & & \multicolumn{2}{|c|}{$<0.0008^{*}$} & \multicolumn{2}{|c|}{$<0.0001 *$} \\
\hline \multirow[t]{2}{*}{$\begin{array}{c}\text { Type of } \\
\text { Restoration }\end{array}$} & $\begin{array}{c}\text { Fissure Sealant } \\
\text { Resin } \\
\text { Amalgam } \\
\text { SSC }\end{array}$ & $\begin{array}{c}349(84.5 \%) \\
28(54.9 \%) \\
63(63.0 \%)\end{array}$ & $\begin{array}{l}140(25.0 \%) \\
21(41.2 \%) \\
27(27.0 \%)\end{array}$ & $\begin{array}{c}29(5.2 \%) \\
2(3.9 \%) \\
10(10.0 \%)\end{array}$ & $\begin{array}{c}296(52.4 \%) \\
23(45.1 \%) \\
61(59.8 \%)\end{array}$ & $\begin{array}{c}269(47.6 \%) \\
28(54.9 \%) \\
41(40.2 \%)\end{array}$ & $\begin{array}{c}359(74.0 \%) \\
25(59.5 \%) \\
61(68.6 \%)\end{array}$ & $\begin{array}{c}126(25.9 \%) \\
17(40.4 \%) \\
28(31.5 \%)\end{array}$ \\
\hline & $P$ value & & $<0.0001 *$ & & \multicolumn{2}{|c|}{0.2271} & \multicolumn{2}{|c|}{0.1265} \\
\hline & $\begin{array}{l}\text { Direct Pulp } \\
\text { Capping }\end{array}$ & $8(80.0 \%)$ & $1(10.0 \%)$ & $1(10.0 \%)$ & $4(40.0 \%)$ & $6(60.0 \%)$ & $9(100.0 \%)$ & 0 \\
\hline $\begin{array}{l}\text { Type of } \\
\text { Pulp }\end{array}$ & $\begin{array}{l}\text { Indirect Pulp } \\
\text { Capping }\end{array}$ & 35 (57.4\%) & $26(42.6 \%)$ & 0 & $33(54.1 \%)$ & $28(45.9 \%)$ & $38(76.0 \%)$ & $12(24.0 \%)$ \\
\hline \multirow[t]{3}{*}{ Therapy } & Pulpotomy & $4(100.0 \%)$ & 0 & 0 & 0 & $4(100.0 \%)$ & $4(100.0 \%)$ & 0 \\
\hline & RCT & 0 & $1(33.3 \%)$ & $2(66.7 \%)$ & $3(100.0 \%)$ & 0 & $1(50.0 \%)$ & $1(50.0 \%)$ \\
\hline & $\mathrm{P}$ value & & $<0.0001 *$ & & \multicolumn{2}{|c|}{0.0512} & \multicolumn{2}{|c|}{0.1812} \\
\hline
\end{tabular}

\section{DISCUSSION}

In this study, a quarter of the children had sound FPMs, which were provided with preventive treatments under GA, such as the application of prophy and fluoride or of fissure sealants on their teeth. The caries-inhibiting effects of fluoride gel [17] and satisfactory placement of sealants was demonstrated in literature. Isolating the FPMs teeth, using resin base materials, employing good operator techniques and protocols, and exploring other methods, should be applied on the FPM to enhance sealant retention [18]. This preventive treatment increases the operative time under general anesthesia.

The vast majority of the children received a combination of both restoration and extraction treatments. This mode of treatment was in agreement with the results of a previous Saudi study that was conducted in Jeddah, where more than two-thirds of the children received both extraction and restoration treatments. This result is higher than those from the last Saudi studies [19], [20]. Although the extraction of the FPM was reported in $17 \%$ of the cases, half of them were described as aggressive and all FPMs were extracted. Only two of the FPMs in $30.8 \%$ of the cases were extracted. With lack of early preventive care and dental home, our study reported that FPMs extraction treatment rate is higher than in other studies, such as the study of FPM extraction in Romanian children [21]. This decision could be explained by the importance of performing FPM extraction at an optimal time, i.e., between $8.5-10$ years for the mandible and as late as 10-11.5 years for the maxilla [23]. Jälevik and Möller reported that favorable spontaneous reduction of space and the development of the permanent dentition could be expected without any extraction intervention in the majority of their cases prior to the eruption of the second permanent molar [23]. Higher children FPM extraction under GA indicated the need for pre comprehensive occlusion assessment by the pediatric dentist to facilitate orthodontic follow up for these cases.

The dental treatment of the FPM under GA at KAMC in our study was following the AAPD recommendation for tooth restoration [24]. Composite resin was used in up to three surface cavities in about half of the FPMs (49\%) in our study. Due to the insufficient evidence to support the use of glass ionomer as a long-term restorative material for permanent teeth, it was not used in treating the FPMs. Although there is strong evidence that dental amalgam is efficacious in the restoration treatment of Class I and Class II cavities in FPMs, our study reported that its use was restricted to less than 5\% of the FPMs treated under GA. On the other hand, in all badly damaged FPMs, more than three surfaces were restored with SSC under GA. The pediatric dentist followed the AAPD recommendation to use preformed metal crowns on the permanent teeth as a semi-permanent restoration strategy for the treatment of severe enamel defects or grossly carious teeth.

Forsyth reported that the length of operation time was influenced by the kind of restorative services applied, the number of treated teeth, and the level of the resident's training background [25]. The operation times in our study was mostly between 60 - $180 \mathrm{~min}$ with longer mean at $120 \mathrm{~min}$ \pm 55 , compared to those reported at $76 \mathrm{~min}( \pm 37 \mathrm{SD})$ in [25] or the mean operation time of $97.9 \mathrm{~min}( \pm 49.9)$ in a previous Saudi multi-center study, with a range of 5 to $224 \mathrm{~min}$ [13]. This higher operation time could be explained by the variations in the patients' ages, as $40 \%$ of previous study sample were below 5 years old. In our study, we report a higher frequency of providing restorative services on the FPMs and the primary dentition and the greater participation allotted to resident training under GA and the involvement of healthier children with mixed dentition, compared to those from the previous studies.

Several factors identified in previous studies had affected the decisions made on which type of FPM dental treatment under GA should be selected, and such factors involved the ages and the medical status of the children [26]-[28]. In our study, age significantly influenced and changed the treatment method, the type of restoration treatment, and pulp therapy, whereas gender and health status had an impact on the treatment method only among the pediatric patients under GA. In a previous Saudi study, healthy children were treated using different approaches in their treatments under GA. This demonstrated the influence of health conditions of the children on the types of treatment given to them [29]. Young children received significantly more preventive treatments, using fissure sealant $(26 \%)$ and restoration $(56 \%)$ due to the early assessment and treatment of their FPMs. FPM extraction was reported in $17 \%$ of the cases under GA, with 
significantly more tooth removal in children under the 8-11year-old group. This could be explained by employing the best age timing for the extraction of the FPMs with advanced caries. The aggressive treatment protocol for teeth with a high risk of developing caries and the uncooperative attitudes of the children could explain the higher FPM extraction rate under GA, especially in 8 to 11-years-old children. Among the $17 \%$ FPM extraction cases under GA, half had four of their FPMs extracted at the same time under GA. This could be explained by the collaborative system for patient comprehensive care between pediatric and orthodontic sections in dental department at KAMC-Jeddah

Our study assessed the frequency and types of FPM procedures under GA, which can guide dentists to better plan for the case. The FPMs high restorative and extraction frequency indicated the early assessment of dental problems concerning the FPMs of children is a must to prevent the initiation and complications caused by dental caries and determine the need to administer aggressive dental treatments as early as possible. On the other hand, with pediatric dentist efforts to effectively estimate and use operation time properly under GA to decrease risk, it is recommended to initiate a prospective study that compares the operation time with or without FPMs treatment. Further study is needed to determine the long-term success of FPM treatment decisions among our children by following the FPMs treatment quality and prognosis for long after GA. The limitation of our study dwells on the nature of its design, being a retrospective study (cross sectional), hospital-based with varies operator level, and is limited to the available documents of the patients. There is a need for prospective studies to confirm the factors affecting the decision making of FPM extraction under different treatment modalities. Other variables that affect the consequences of FPM extraction among pediatric patients should also be tested.

\section{CONCLUSIONS}

Our study reveals that pediatric dentists' decision for FPMs treatment under general anesthesia at KAMC was following AAPD guideline and its frequency was mostly restorative followed by preventive, and the least treatment was extraction treatments. The type of FPMs treatment administered on children should consider their ages, gender, and health conditions. KAMC pediatric dentist efforts should be directed to initiate more preventive programs and effective dental home care utilization at PHC centers. All preventive efforts should be initiated to prevent FPM caries as early as possible. FPM extraction among children treated under GA should be planned carefully by the pediatric dentist to facilitate orthodontic follow up for these cases.

\section{LIST OF ABBREVIATIONS}

GA: general anesthesia; FPM: first primary molar; KAMC: King Abdulaziz Medical City; SSC: stainless steel crown; SD: standard deviation; RCT: root canal treatment; DMFT: Decayed, Missing, and Filled Teeth; AAPD: American Academy of Pediatric Dentistry.

\section{ETHics APPROVAl AND CONSENT TO PARTICIPATE}

This study was approved by the King Abdullah International Medical Research Center, the King Saud bin Abdulaziz University for Health Sciences, and the National Guard Health Affairs through Protocol No. RSS18/-41/J and Approval No. RYD-18-417780-132823.

\section{ACKNOWLEDGMENT}

We would like to thank Dr. Duaa Olwi our epidemiologist, Dr. Ramish Vishwakarama for data analysis, the pediatric dentistry section at KAMC-Jeddah and the Research Summer Student team at KSAU-HS and KAIMRC for the great help in the conducting this study.

\section{FUNDING}

This study did not receive any funding.

\section{CONFLICT OF INTEREST}

Authors declare that they do not have any conflict of interest.

\section{REFERENCES}

[1] Saber AM, Altoukhi DH, Horaib MF, El-Housseiny AA, Alamoudi NM, Sabbagh HJ. Consequences of early extraction of a compromised first permanent molar: A systematic review. BMC Oral Health. 2018; 18(1): 59

[2] Alkhadra T. A systematic review of the consequences of early extraction of first permanent first molar in different mixed dentition stages. J Int Soc Prev Community Dent. 2017; 7(5): 223.

[3] Jamjoom MM, Al-Malik MI, Holt RD, El-Nassry A. Dental treatment under general anaesthesia at a hospital in Jeddah, Saudi Arabia. Int $J$ Paediatr Dent. 2001;11(2): 110-116.

[4] Dupã PE. Extraction of the first permanent molars and the intervention of the orthodontics specialist. Romanian Dent J. 2008; 54: 66-72.

[5] Bartella AK, Lechner C, Kamal M, Steegmann J, Hölzle F, Lethaus B. The safety of paediatric dentistry procedures under general anaesthesia: Five-year experience of a tertiary care centre. Eur J Paediatr Dent. 2018; 19(1): 44-48.

[6] Al-Emran S. Prevalence of tooth loss in Saudi Arabian school children: An epidemiological study of Saudi male children. Saudi Dent J. 1990; 2: $137-140$.

[7] Saber AM, Altoukhi DH, Horaib MF, El-Housseiny AA, Alamoudi NM, Sabbagh HJ. Consequences of early extraction of compromised first permanent molar: a systematic review. BMC Oral Health. 2018; 18(59):1-15

[8] Gill DS, Lee RT, Tredwin CJ. Treatment planning for the loss of first permanent molars. Dent Update. 2001; 28(6): 304-8

[9] Al-Malik MI, Rehbini YA. Prevalence of dental caries, severity, and pattern in age 6 to 7 -year-old children in a selected community in Saudi Arabia. J Contemp Dent Pract. 2006; 7(2): 46-54.

[10] Al-Namankany A. Assessing dental anxiety in young girls in KSA. Journal of Taibah University Medical Sciences. 2018; 13(2): 123-128

[11] Baakdah RA, Turkistani JM, Al-Qarni AM, Al-Abdali AN, Alharbi HA, Bafaqih JA, et al. Pediatric dental treatments with pharmacological and non-pharmacological interventions: a crosssectional study. BMC Oral Health. 2021; 21: 186.

[12] Farsi N, Baakdah R, Boker A, Almushayt A. Postoperative complications of pediatric dental general anesthesia procedure provided in Jeddah hospitals, Saudi Arabia. BMC Oral Health. 2009; $9(6)$.

[13] Ba-akdah R, Farsi N, Boker A, Al Mushayt A. The use of general anesthesia in pediatric dental care of children at multi-dental centers in Saudi Arabia. Int J Pediatr Dent. 2008; 33(2): 147-53.

[14] Atan S, Ashely P, Gilthorpe MS, Scheer B, Mason C, Roberts G. Morbidity following dental treatment of children under intubation general anesthesia in a day-stay unit. Int J Pediatr Dent. 2004; 14: 9 16. 
[15] Ogg TW, McDonald IA, Jennings RA, Morrison CG. Day-case dental anesthesia: evaluation of three methods of anesthesia. Br Dent J. 1983; 155: 14-17.

[16] Holt RD, Chidiac RH, Rule DC. Dental treatment for children under general anesthesia in day care facilities at a London dental hospital. $\mathrm{Br}$ Dent J. 1991; 170: 262-266.

[17] Marinho VCC, Worthington HV, Walsh T, Chong LY. Fluoride gels for preventing dental caries in children and adolescents. Cochrane Database Syst. Rev. 2015; (6).

[18] Azarpazhooh A, Main PA. Pit and fissure sealants in the prevention of dental caries in children and adolescents: A systematic review. $J$ Can Dent Assoc. 2008; 74(2): 171-177.

[19] Osuji OO, Assery MK. The dental treatment of children under general anaesthesia at a hospital in Taif, Saudi Arabia. SDJ. 2005; 17: 120125

[20] Jamjoom MM, Al-Malik MI, Holt RD, El-Nassry A. Dental treatment under general anaesthesia at a hospital in Jeddah, Saudi Arabia. Int $J$ Paediatr Dent. 2001; 11: 110-116.

[21] Rãducanu AA, Feraru V, Herteliu C, Rãducanu MA. Prevalence of loss of permanent first molars in a group of Romanian children and adolescents. OHDMBSC. 2009; VIII(3): 3-10.

[22] 2Bourbonnais R. Econométrie, 5th ed. Paris: Dunod; 2005.

[23] Jälevik B, Möller M. Evaluation of spontaneous space closure and development of permanent dentition after extraction of hypomineralized permanent first molars. Int J Paediatr Dent. 2007; 17: $328-335$.

[24] American Academy of Pediatric Dentistry (AAPD). Policy and guideline. Pediatr Restor Dent. 2019: 340-352.

[25] Forsyth AR, Seminario AL, Scott J, Berg J, Ivanova I, Lee H. General anesthesia time for pediatric dental cases. Pediatr Dent. 2012; 34(5): $129-135$.

[26] Mason C, Holt RD, Rule DC. The changing pattern of day-care treatment for children in a London dental teaching hospital. Br Dent $J$. 1995; 179: 136-140.

[27] Wong FSL, Fearne JM, Brook AH. Planning future general anaesthetic services in pediatric dentistry on the basis of evidence: an analysis of children treated in the Day Stay Centre at the Royal Hospitals NHS Trust, London, between 1985-1995. Int Dent J. 1997; 47: 285-292.

[28] Haubek D, Fuglsang M, Poulsen S, Rolling, I. Dental treatment of children referred to general anaesthesia association with country of origin and medical status. Int J Paediatr Dent. 2006; 16: 239-246.

[29] Al-Ogayyel S, Al-Haj AS. Comparison of dental treatment performed under general anaesthesia between healthy children and children with special health care needs in a hospital setting, Saudi Arabia. J Clin Exp Dent. 2018; 10(10): e963-9. 